\title{
ERRATA TO “THE NONTRIVIALITY OF THE FIRST RATIONAL HOMOLOGY GROUP OF SOME CONNECTED INVARIANT SUBSETS OF PERIODIC TRANSFORMATIONS"
}

\author{
A. ASSADI AND D. BURGHELEA
}

The reference to [K1] in the proof of Theorem A should be replaced by [K, S] and $[\mathbf{R}]$; the latter papers combine to give a correct proof for the result stated in [K1]. The proof of Theorem $\mathrm{A}$ as given works for $n \neq 4$. The case $n=4$ follows from $n=5$ by applying Theorem $\mathrm{A}$ to $M \times \mathbf{R}$ (equipped with action $\tilde{\mu}(g ;[m, t])=$ $[\mu(g, m), t]$.

The hypotheses of Theorem $\mathrm{C}$ should include the following:

(a) $M^{n+2}$ is a closed manifold.

(b) If $G=Z_{m}$ in part (1) or $G_{m, r}$ in part (2), then $G$ acts trivially on $\pi_{1}(M)$.

These hypotheses are used in the proof.

We thank D. Ruberman for pointing out the insufficiency and inaccuracy of reference [K1], and A. Edmonds for pointing out the obvious failure of Theorem C without the additional hypotheses.

The following misprints should be corrected:

p. 705, line 6 from bottom: $\tilde{H}_{*}\left(M^{\mathbf{Z}_{m}} ; Z_{m}\right)$ should be $H_{*}\left(\tilde{M}^{\mathbf{Z}_{m}} ; Z_{m}\right)$.

p. 705 , line 5 from bottom: $H\left(M ; Z_{m}\right)$ should be $H_{*}\left(\tilde{M} ; Z_{m}\right)$.

p. 706, line 2: $\tilde{P}_{i} \rightarrow K(\pi, 1)$ should be $P_{i} \rightarrow K(\pi, 1)$.

p. 707, line 8: $M^{\mathbf{z}_{n}}$ should be $M^{\mathbf{Z}_{r}}$.

\section{REFERENCES}

[A, S] A. Assadi and D. Burghelea, The nontriviality of the first rational homology group of some connected invariant subsets of periodic transformations, Proc. Amer. Math. Soc. 88 (1983), 701-707.

[K, S] R. Kirby and L. Siebenmann, Normal bundles for codimension 2 locally flat embeddings, Proc. Topology Conf. (Park City, Utah, 1974), Lecture Notes in Math., vol. 438, Springer, New York, 1975, pp. 310-324.

[R] D. Ruberman, Locally flat surfaces in four manifolds have normal bundles (to appear).

Department of Mathematics, University of Virginia, Charlottesville, Virginia 22903

Department of Mathematics, Ohio State University, Columbus, Ohio 43210

Received by the editors February 14, 1984. 